\title{
Adult hippocampal neurogenesis: a phenomenon in search of a function
}

\author{
Julian R. Keith
}

Published online: 25 April 2007

(C) Springer Science+Business Media, LLC 2007

During the 1960s and 1970s, Joseph Altman and his colleagues presented evidence for the radical idea that adult mammalian brains produce new neurons. Altman's group studied brains from rats and cats that were administered tritiated thymidine, a compound that is incorporated into DNA during mitosis, and when they performed postmortem autoradiography, they observed neurons decorated with "thymidine grains," especially in the subventricular zone and the dentate gyrus of the hippocampus. However, Altman's data did not sway many neuroscientists from the view that mammalian neurogenesis is completed before birth. Skepticism about adult neurogenesis was warranted at the time because thymidine-labeled neurons can be difficult to distinguish morphologically from glia, mitotic figures are not observed in mature neurons (the concepts of stem cells and neuronal progenitor cells were not yet available), and DNA repair could potentially explain the incorporation of tritiated thymidine into neurons. Moreover, when several research groups reported in the 1980s that they failed to observe evidence of neurogenesis in the brains of adult primates, it appeared that the concept of adult neurogenesis would not be relevant to the problem of understanding the primate brain.

The table has turned and now research on this adult mammalian neurogenesis is flourishing. Technical obstacles to identifying new neurons have been solved by the development of immunohistochemical markers for cell phenotyping and confocal microscopy. We now know that neurons are being born continuously in the hippocampus and subventricular zone of adult mammalian brains. Of course, many important questions about adult neurogenesis

\section{J. R. Keith $(\bowtie)$}

Department of Psychology, The University of North Carolina,

Wilmington, 601 South College Road,

Wilmington, NC 28403, USA

e-mail: keithj@uncw.edu remain. However, no issue is more pivotal to this enterprise than whether new neurons participate in any informationprocessing functions. Definitive answers to that question are more elusive than the original questions about whether adult neurogenesis occurs. The articles that follow evaluate the current state of the evidence related to the question of whether new neurons contribute to the functionality of the adult brain.

Heather Cameron and Brian Christie have contributed an article entitled "Do new neurons have a functional role in the adult hippocampus?" Cameron and Christie argue that enough new neurons are born in the hippocampus to reasonably expect that they would have an impact the hippocampal system's circuitry and that various techniques that have been used to arrest neurogenesis also impair certain aspects of learning and memory. While no individual study alone is sufficient, Cameron and Christie argue that, collectively, the data solidly point to the conclusion that new neurons must play a role in hippocampus function.

In the second article, entitled "Adult neurogenesis: a reality check", Helena Frielingsdorf and Georg Kuhn argue that the notion that new neurons contribute to normal functioning in the healthy adult brain or can serve to repair injured brain systems still should be rated as hypothetical, though promising. Frielingsdorf and Kuhn question whether neurogenesis in brain regions other than the hippocampus and the subventricular zone is possible under normal in vivo conditions in the adult brain. The authors also take issue with aspects of the techniques and experimental designs often used to study neurogenesis and challenge interpretations of studies that have attempted to link neurogenesis rates to behavioral performance.

All four authors who contributed to this debate previously have made substantial empirical contributions to the study of adult neurogenesis. Here, they offer two distinctly different, and provocative, appraisals of the meaning of those data. 